\title{
Age and gender-specific distribution of metabolic syndrome components in East China: role of hypertriglyceridemia in the SPECT-China study
}

Boren Jiang ${ }^{\dagger}$, Yanjun Zheng ${ }^{\dagger}$, Yingchao Chen, Yi Chen, Qin Li, Chunfang Zhu, Ningjian Wang, Bing Han, Hualing Zhai, Dongping Lin and Yingli Lu* (D)

\begin{abstract}
Background: Chinese population are experiencing remarkably changes of economic and cultural environments. The present study was to examine the prevalence of metabolic syndrome (MetS) by age between genders and to investigate the current characteristics of MetS and its components in China.

Methods: SPECT-China is a population-based cross-sectional survey on Chinese adults aged $\geq 18$ years in East China. A total of 10,441 Chinese residents participated in anthropometric and laboratory measurements. Of these, 9969 subjects (females, 5868) were eligible for the data analysis reported here. Estimates of the prevalence of MetS and its components were calculated. Presence of MetS was defined based on the IDF/AHA harmonized criteria. MetS z-score was calculated to evaluate the degree of total metabolic disorder.

Results: The age-standardized prevalence of the metabolic syndrome was 22.0\% (21.9\% in men and 22.0\% in women). Unlike the continuous MetS rise with age in females, the MetS prevalence in males remained stable among 46-55, 56-65 and $>65$ yrs. age groups $(31.2 \%, 31.4 \%, 32.5 \%, p=0.538)$. In the five components of MetS, contrary to the elevated BP and BG disorders, the frequency of TG disorders decreased with age in males (46.6\%(46-55 yrs), 37.2\% (56-65 yrs), 27.7\%(> 65 yrs), $p<0.001)$. Multivariable logistic regression showed that in males, more TG disorders were associated with higher BMI, higher educational level and current nonsmoker. In the MetS subjects, the 3-factor combinations which included TG disorders decreased with age in both genders. The whole metabolic profile became better in older male MetS subjects, which was opposite to the female.
\end{abstract}

Conclusion: Our results showed a distinct age-related prevalence of MetS between genders in dramatically changed China, in which the TG disorders played an important role. More targeted measures need to be taken to meet the serious challenges of metabolic diseases.

Trial registration: ChiCTR-ECS-14005052, Survey on Prevalence in East China for Metabolic Diseases and Risk Factors (SPECT-China).

Keywords: Metabolic syndrome, Hypertriglyceridemia, Cross-sectional design, Gender, Chinese

\footnotetext{
* Correspondence: luyingli2008@126.com

${ }^{\dagger}$ Equal contributors

Department of Endocrinology and Metabolism, Shanghai Ninth People's

Hospital, Shanghai JiaoTong University, School of Medicine, Shanghai

200011, China
}

(c) The Author(s). 2018 Open Access This article is distributed under the terms of the Creative Commons Attribution 4.0 International License (http://creativecommons.org/licenses/by/4.0/), which permits unrestricted use, distribution, and reproduction in any medium, provided you give appropriate credit to the original author(s) and the source, provide a link to the Creative Commons license, and indicate if changes were made. The Creative Commons Public Domain Dedication waiver (http://creativecommons.org/publicdomain/zero/1.0/) applies to the data made available in this article, unless otherwise stated. 


\section{Background}

Metabolic syndrome (MetS) is defined as a cluster of metabolic abnormalities including central obesity, hypertension, high plasma triglycerides, decreased high density lipoprotein (HDL) cholesterol and glucose intolerance. Having any three of the five abnormal indicators above has been defined as MetS [1]. It has been shown that MetS increases the risk for atherosclerotic cardiovascular disease (ASCVD) and diabetes mellitus [2,3], which are major contributors to morbidity and mortality all over the world [4-6], including China [7].

Epidemiologic studies have demonstrated differences in MetS prevalence by geography, age, gender and ethnicity. Taking age as an example, from NHANES III in the US [8], a marked increase in prevalence of the MetS from 20 years of age through the sixth decade of life was noted for men and through the seventh decade for women. However, prevalence declined in the eighth decade of life. A different pattern was observed from the Norwegian HUNT II Study [9]. In this study, the MetS prevalence increased linearly with age into the ninth decade of life. The MetS prevalence and components also markedly varied with aging in the same group of population. From 1988 to 1994 to 2007-2012, the prevalence of MetS increased from 25.3\% to $34.2 \%$ in the US [10]. Our previous data showed the unstandardized prevalence of MetS elevated from 29.65\% (2005) to $45.49 \%$ (2014) from a community study in China [11]. Although the underlying causes of these variations were not clearly demonstrated yet, they usually have been attributed to differences in genetic and environmental factors such as dietary patterns, activity, stress levels and socioeconomic status $[12,13]$.

Chinese people are experiencing remarkably changes of economic and cultural environments, such as factors related to rapid nutritional transition, rural-to-urban migration, adoption of a sedentary lifestyle and mental stress $[14,15]$, which may contribute to the progression of metabolic syndrome. It is crucial to get undated knowledge about the characteristics of metabolic syndrome in China, which can help design specific preventative and therapeutic strategies. Several previous epidemiologic studies have displayed important information about the national or regional prevalence of MetS in China [16-20]. However, detailed data about the prevalence and characteristics of MetS by age in different genders are scarce.

The present study was to examine the prevalence of MetS by age between genders and to investigate the current characteristics of MetS and its components in China. We aimed to get a better understanding of MetS for future specific prevention and treatment in China.

\section{Methods}

\section{Study design and population}

SPECT-China (ChiCTR-ECS-14005052, www.chictr.org.cn) is a population-based cross-sectional survey on prevalence of metabolic diseases and risk factors in East China, which is made up of Shanghai and 7 provinces with a population of approximately 395 million in 2011, accounting for $29.2 \%$ of people in China. Detailed sampling information was described in a previous study [21]. In brief, the study was conducted from February 2014 to December 2015. Twenty-two residential sites in Shanghai, Zhejiang, Jiangxi, Jiangsu and Anhui Province were selected using a stratified and cluster sampling method. Chinese citizens $\geq 18$ years old who had lived in their current area for $\geq 6$ months were selected. We excluded subjected with severe communication problems, acute illness or who were unwilling to participate. A total of 10,441 subjects were enrolled in SPECT-China study finally. This study was conducted according to the principles laid down in the Declaration of Helsinki of 1975, was approved by the Ethics Committee of Shanghai Ninth People's Hospital, Shanghai JiaoTong University School of Medicine. All patients to be included signed the informed consent. For participants who were illiterate, we obtained written informed consent from their proxies. After excluding individuals who had missing MetS components (WC, BP, FBG, TG or HDL) data or were pregnant or had active malignant tumors ( $n=472)$, a total of 9969 subjects (females, 5868) were finally analyzed.

\section{Demographic, anthropometric and laboratory measurements}

Subjects were interviewed face-to-face to complete pretested questionnaires covering sociodemographic information, medical history of coronary heart diseases, diabetes, hypertension and lipid disorders.

Marital status was recorded in four categories: single, married/co-habiting, divorced, and widowed. Education level, determined on the basis of the highest level achieved, was classified into three categories: primary school or below, middle school, high school or above. Occupational position was categorized as non-manual, manual and self-employed. Cigarette smoking was assessed by self-report from the question "Do you smoke cigarettes now?" (yes/ no). Alcohol consumption was assessed based on how often participants consumed wine, beer, or hard liquor, as follows: less or equal than one drinks per week (none current drinker), and two drinks or more per week (current drinker).

Height and weight were measured to the nearest $0.5 \mathrm{~cm}$ and $0.1 \mathrm{~kg}$, respectively, with the participants wearing 
light-weight clothing and without shoes. Body mass index (BMI) was calculated as weight $(\mathrm{kg})$ divided by height squared (m2). WC was measured on standing participants midway between the lower edge of the costal arch and the upper edge of the iliac crest using a non-elastic tape (to the nearest $0.5 \mathrm{~cm}$ ). All anthropometric measurements were taken in duplicate and the averages of these measurements were used in the analyses.

Resting systolic and diastolic BP was measured three times at 1-min intervals using a standard mercury sphygmomanometer after a 5-min rest. The average of the second and the third readings were used in the analyses.

Fasting venous blood samples were collected in the morning after at least $8 \mathrm{~h}$ of fasting. The samples for plasma glucose test were collected into vacuum tubes with anticoagulant sodium fluoride and centrifuged on the spot in $1 \mathrm{~h}$ after collection. Blood samples were shipped in dry ice to a central laboratory within 2-4 h of collection. FPG, HDL-C and TG were analyzed enzymatically using an autoanalyzer. All laboratory equipment was calibrated, and blinded duplicate samples were used for these analyses.

\section{Definition of metabolic syndrome}

MetS was defined based on the IDF/AHA harmonized criteria [1]. Thus, positive diagnosis of the syndrome was established when at least three of the following were present: (1) Waist circumference $\geq 90 \mathrm{~cm}$ in men or $\geq 80 \mathrm{~cm}$ in women [22]; (2) HDL cholesterol $<1.0 \mathrm{mmol} / \mathrm{L}$ in men or $<1.3 \mathrm{mmol} / \mathrm{L}$ in women; (3) Serum TGs $\geq 1.7 \mathrm{mmol} / \mathrm{L}$ (4) Serum glucose level $\geq 5.6 \mathrm{mmol} / \mathrm{L}$; (5) Blood pressure $\geq 130 / 85 \mathrm{mmHg}$. Treatment with anti-hypertensive, hypoglycemic or lipid-lowering drugs was considered as alternate indicators of the latter three components. Agestandardized incidence rates of MetS were calculated using direct standardization with population composition of the Sixth National Population Census of China (2010). The 1999 World Health Organization (WHO) diagnostic criteria were used to diagnose Diabetes Mellitus [23]. A subject with SBP or DBP $\geq 140 / 90 \mathrm{mmHg}$ is considered hypertension by WHO/ISH 1999 criteria [24].

MetS z-score was calculated. It takes into account continuous changes in each component, representing the score of continuous risk for MetS [25]. For each risk factor, a z-score was calculated (individual value sample mean/standard deviation of the sample). For the blood pressure, we used the MAP $(2 / 3 \mathrm{DBP}+1 / 3$ SBP) of for calculating the score. Total score = waist $\mathrm{Z}$ score $+\mathrm{BP} \mathrm{Z}$ score + glucose $\mathrm{Z}$ score + HDL-C Z score + triglycerides $Z$ score. A lower risk score is indicative of a better metabolic profile.

\section{Statistical analysis}

All analyses were stratified by sex. We grouped participants by age as follows: $18-45$ yrs. $(n=1044), 46-55$ yrs. $(n=1088), 56-65$ yrs. $(n=1130)$ and $>65$ yrs. $(n=839)$. Continuous variables are presented as means (SD, normal distributions) and median (interquartile range, skewed distributions) and proportions were calculated for discrete variables. To test differences of characteristics among different age groups, the 1-way ANOVA was applied for continuous data (FBG and TG were normalized by inverse and logarithmic transformation) and the Pearson chi-square test with linear by linear association was performed for categorical variables. Univariate and multivariable logistic regression analysis was performed to determine odds ratios (ORs) and 95\% confidence intervals (CIs) for TG disorder and conventional risk factors. In the process of analyzing the frequency of components combination of MetS, all kinds of 3-factor combinations were counted. The actual frequency of one 3 -factor combination is equal to the sum of the number of this combination in 3-factor group and the number of 4-factor and 5-factor group including this 3-factor combination (The theoretical frequency of total random 3 parameters combination equal to (total 3-factor frequency)*1 plus (total 4-factor frequency)*4 plus (total 5factor frequency)*5). Data were analyzed using SPSS software version 22.0 for Mac, with the significance level set at $p<0.05$ for all analyses.

\section{Results}

A total of 9969 subjects (females, 5868) with a mean \pm SD age of $53.34 \pm 12.99$ years were analyzed in this study. All participants were of Han origin. From Table 1, compared with males, the female participants had lower educational level, more manual worker, less current tobacco smokers, less alcohol drinkers and less recognized hypertensive subjects. The living area, marital status, history of DM and CVD were not statistically different between men and women. The crude MetS prevalence, using the IDF/AHA harmonized criteria, were significantly higher in females than males $(32.3 \%$ vs $28.5 \%, P<0.001)$. However, the agestandardized MetS prevalence showed no difference between genders ( $21.9 \%$ vs $22.0 \%, P>0.05)$.

The prevalence of MetS and its components between age groups $(18-45,46-55,56-65,>65$ yrs) were shown in Fig. 1. In female subjects, the frequency of MetS rose rapidly with age increase $(10.4 \%, 29.9 \%, 44.9 \%, 54.9 \%$, respectively, $P<0.001)$. In males, although the MetS frequency was significantly higher in older subjects ( $>45$ yrs) than $18-45$ yrs. age group, there were no differences among 46-55, 56-65 and $>65$ yrs. age groups, which meant the MetS prevalence did not go up with age in males. In 18-45 yrs. age group, the frequency of MetS was higher in males than females 
Table 1 Characteristics of study population by gender

\begin{tabular}{|c|c|c|c|c|}
\hline & Total $(n=9969)$ & Male $(n=4101)$ & Female $(n=5868)$ & $P$ value \\
\hline Age (year) & 53.34(12.99) & $54.05(13.09)$ & $52.85(12.90)$ & $<0.001$ \\
\hline BMI $(\mathrm{kg} / \mathrm{m} 2)$ & $24.53(3.52)$ & $24.86(3.35)$ & $24.30(3.62)$ & $<0.001$ \\
\hline Living area, \% & & & & $>0.05$ \\
\hline Rural & 58.4 & 59.1 & 57.8 & \\
\hline Urban & 41.6 & 40.9 & 42.2 & \\
\hline Educational level, \% & & & & $<0.001$ \\
\hline High school or above & 20.6 & 25.8 & 16.9 & \\
\hline Middle school & 44.1 & 47.1 & 42 & \\
\hline Primary school or below & 35.3 & 27.1 & 41.1 & \\
\hline Marital status, $\%$ & & & & $>0.05$ \\
\hline Married/co-habiting & 93.8 & 93.9 & 93.8 & \\
\hline Single/divorced/widowed & 6.2 & 6.1 & 6.2 & \\
\hline Occupational position, \% & & & & $<0.001$ \\
\hline Manual & 52.7 & 49.1 & 55.2 & \\
\hline Non-manual & 34.8 & 35.5 & 34.2 & \\
\hline Self-employed & 12.6 & 15.4 & 10.6 & \\
\hline Current smoking status, $\%$ & 21.2 & 48.2 & 2.3 & $<0.001$ \\
\hline Current drinking status, $\%$ & 54.1 & 68.1 & 44.3 & $<0.001$ \\
\hline History of hypertension, \% & 26.1 & 28.7 & 24.3 & $<0.001$ \\
\hline History of DM, \% & 7.3 & 7.6 & 7.1 & $>0.05$ \\
\hline History of CVD, \% & 5.7 & 5.3 & 6.1 & $>0.05$ \\
\hline Metabolic syndrome, \% (unstandardized) & 30.8 & 28.5 & 32.3 & $<0.001$ \\
\hline Metabolic syndrome, \% (standardized) & 22.0 & 21.9 & 22.0 & $>0.05$ \\
\hline
\end{tabular}

Data were showed as means \pm SD or percentages (\%). BMI body mass index, DM diabetes mellitus, CVD Cardiovascular diseases

(19.4\% vs $10.4 \%, P<0.001)$. In older $56-65$ and $>65$ yrs. age groups, the frequency of MetS became lower in males than female counterparts (31.4\% vs $44.9 \%, 32.5 \%$ vs $54.9 \%$, both $P<0.001)$. To explore the underlying factors influencing MetS prevalence, the frequency of MetS component abnormalities (among 46-55, 56-65, > 65 yrs. age groups) were analyzed. In females, all of the MetS components showed increased abnormal frequencies with age except HDL, which kept stable across different age groups. In males, the FBG and BP abnormalities displayed similar ]trend as that in females and the WC and HDL abnormalities kept stable. However, the abnormal TG frequencies decreased with age $(46.6 \%, 37.2 \%, 27.7 \%$, respectively, $P<0.001)$. The values of MetS components showed similar trends as their abnormal frequencies (Table 2). These results suggest the non-increase of male MetS prevalence with age could be attributed to the improved TG disorders.

We conducted a multiple logistic regression analysis of TG disorders and its associated factors in subjects aged older than 45 yrs. (Table 3, factors information see Additional file 1: Table S1). In males, individuals who have higher educational level or higher BMI were more likely to have TG disorders. Current smoking status, aged 56 yrs. or older were found to be inversely associated with having TG disorders. In females, aged 56 yrs. or older, higher BMI and manual occupation were associated with higher TG disorders.

In view of the opposite alteration of $\mathrm{BP}, \mathrm{BG}$ and TG disorders with age increase in males, we further analyzed the change of metabolic disorder spectrum in the MetS population among age groups. From Table 4, in male MetS, the frequency of TG disorder was getting less and BP disorder was getting more with older age. The frequency of abnormal components changed from ( $\mathrm{TG}>\mathrm{BP}>\mathrm{WC}>\mathrm{FBG}>\mathrm{HDL}$ ) to (BP $>\mathrm{FBG}>\mathrm{WC}>\mathrm{TG}>\mathrm{HDL})$. In female MetS subjects, the markedly increased frequency of abnormal FBG and BP, together with decreased rate of abnormal TG and HDL with age, also dramatically changed the metabolic disorder spectrum. To quantify the total impact of metabolic disorders, we calculated the MetS $\mathrm{z}$-score and found that the whole metabolic profile got better with older age in males (56-65 yrs. vs $46-55$ yrs., > 65 yrs. vs $46-55$ yrs., both 

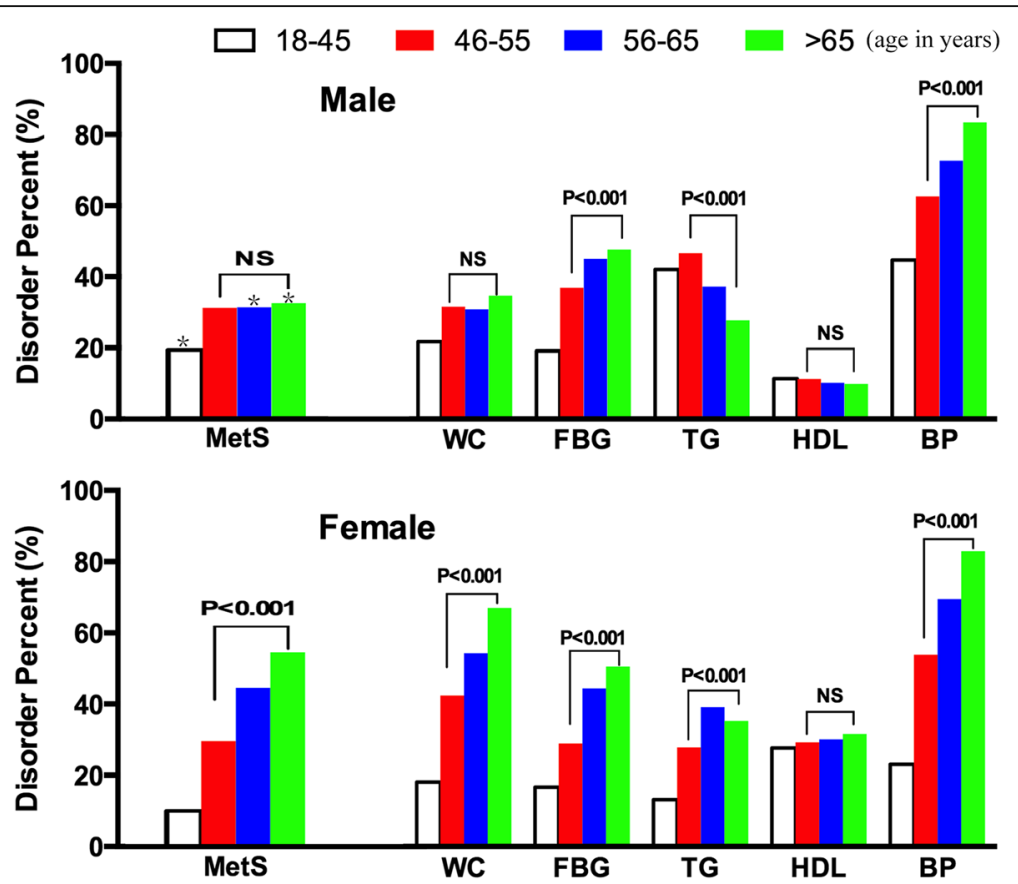

Fig. 1 Frequency of MetS and each MetS component according to gender and age group. ${ }^{*}, P<0.001$, compare male with female MetS from same age group. $P$ value: linear by linear association of Chi-Square Tests among $46-55,56-65$ and $>65$ yrs. age group. NS indicates non-significance

$P<0.05)$, but in females the whole metabolic profile showed worse change (56-65 yrs. vs $46-55$ yrs., $P<0.05$ ).

The clustering of various components was further assessed (Additional file 2: Table S2). Using 3 parameters as a combination, in males, we found the combinations which included BP and excluded TG (WC + BP + HDL, $\mathrm{WC}+\mathrm{BP}+\mathrm{FBG}, \mathrm{HDL}+\mathrm{BP}+\mathrm{FBG}$ ) accounted for more proportion in 56-65 and $>65$ than $46-55$ yrs. age group (29.5\%, $34.2 \%$ vs $24.1 \%$, both $P<0.05)$. On the contrary, the combinations which included TG and excluded BP (WC + TG + HDL, WC + TG + FBG, HDL + TG + FBG) took less proportion in $56-65$ and $>65$ than $46-55$ yrs. age group $(21.5 \%, 18.2 \%$ vs $25.0 \%$, both $P<0.05)$. In females the similar trend did exist, but to a less extent (Fig. 2).

\section{Discussion}

In this study, we investigated the age-related MetS prevalence between genders from a cross-sectional survey in East China. The results showed that unlike the continuous MetS rise with age in females, the MetS prevalence in males remained stable among aged 46 yrs. or older age groups, which could be attributed to the improved TG disorders. In the MetS subjects, the frequency of abnormal components also markedly changed, in which TG disorder became less frequent and BP disorder became more frequent with advancing age. The whole metabolic profile got better in older male MetS subjects, which was opposite to the female. To the best of our knowledge this is the first study to specifically describe the different pattern of MetS prevalence with age between genders and note the important role of TG played in this course in China. Our results provided a better understanding of the current characteristics of MetS in China for future specific prevention and treatment.

The MetS incidence kept stable in aged 46 yrs. or older age groups. This pattern was distinct from other population. From NHANES 1999-2000, the prevalence of MetS continued to rise among 20-39, 40-59 and $\geq 60$ yrs. age groups. Another analysis investigated prevalence by decade of age from NHANES 2003-2006 and found the prevalence rose until aged 60-69 yrs. in both genders, and declined thereafter [26-28]. Data from 10 European countries also showed the MetS prevalence increased with advancing age (from 3.7 in the group aged $20-29$ yrs. to more than $30 \%$ in the subjects 70 yrs. and older) [29, 30]. In a study from Saudi population, the prevalence of MetS increased uniformly with advancing age until aged 60-65 yrs. in both male and female [31]. However, a couple of previous studies from China displayed similar prevalence pattern to ours. Results from residents living in Northeast China rural area [17] showed the MetS prevalence kept stable among 35-44, 45-54 and 55-64 yrs. age groups. Another study from Northeast China even showed declining prevalence among 45-54, 55-64 and $\geq 65$ yrs. age groups in males [19]. Above evidence suggest there might be a 


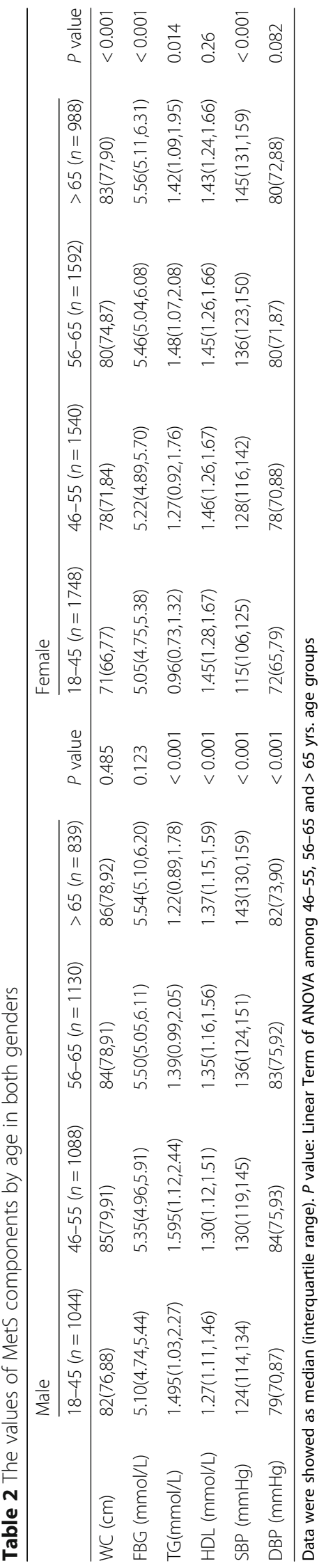


Table 3 Associated factors of TG disorders in multivariate logistic regression analysis

\begin{tabular}{|c|c|c|c|c|}
\hline \multirow[b]{2}{*}{ Variable } & \multicolumn{2}{|l|}{ Male } & \multicolumn{2}{|l|}{ Female } \\
\hline & Odds ratio $(95 \% \mathrm{Cl})$ & $P$ value & Odds ratio $(95 \% \mathrm{Cl})$ & $P$ value \\
\hline \multicolumn{5}{|l|}{ Age (years) } \\
\hline $46-55$ & 1.0 & & 1.0 & \\
\hline $56-65$ & $0.798(0.651,0.979)$ & 0.03 & $1.568(1.306,1.882)$ & $<0.001$ \\
\hline$>65$ & $0.632(0.499,0.800)$ & $<0.001$ & $1.413(1.140,1.751)$ & 0.002 \\
\hline BMI (kg/m2) & $1.2(1.167,1.234)$ & $<0.001$ & $1.146(1.121,1.171)$ & $<0.001$ \\
\hline \multicolumn{5}{|l|}{ Living area } \\
\hline Urban & 1.0 & & 1.0 & \\
\hline Rural & $1.069(0.865,1.321)$ & 0.535 & $1.007(0.846,1.197)$ & 0.939 \\
\hline \multicolumn{5}{|l|}{ Educational level } \\
\hline Primary school or below & 1.0 & & 1.0 & \\
\hline Middle school & $1.252(1.009,1.552)$ & 0.041 & $1.006(0.832,1.216)$ & 0.952 \\
\hline High school or above & $1.566(1.118,2.194)$ & 0.009 & $1.041(0.727,1.490)$ & 0.827 \\
\hline \multicolumn{5}{|l|}{ Marital status } \\
\hline Married/co-habiting & 1.0 & & 1.0 & \\
\hline Single/divorced/widowed & $1.59(0.947,2.668)$ & 0.079 & $1.019(0.743,1.399)$ & 0.905 \\
\hline \multicolumn{5}{|l|}{ Occupational position } \\
\hline Manual & 1.0 & & 1.0 & \\
\hline Non-manual & $0.893(0.670,1.189)$ & 0.438 & $0.676(0.491,0.930)$ & 0.016 \\
\hline Self-employed & $0.871(0.688,1.103)$ & 0.253 & $0.896(0.732,1.097)$ & 0.289 \\
\hline \multicolumn{5}{|l|}{ Current smoking status } \\
\hline Yes & 1.0 & & 1.0 & \\
\hline No & $1.192(1.003,1.416)$ & 0.046 & $1.065(0.669,1.695)$ & 0.79 \\
\hline \multicolumn{5}{|l|}{ Current drinking status } \\
\hline Yes & 1.0 & & 1.0 & \\
\hline No & $1.069(0.883,1.293)$ & 0.495 & $1.135(0.974,1.322)$ & 0.105 \\
\hline
\end{tabular}

specific age-related prevalence of MetS in current Chinese population.

In the five components of MetS, the frequency of abnormal FBG, BP, WC and HDL increased or remained stable in older age groups in both genders. Abnormal TG is less frequent with advancing age in males, while more frequent in females, which suggest the opposite TG changes play a key role in the gender-specific prevalence pattern. The decrease of TG with older age in males had ever been reported. An epidemiological survey in China found the percentage of abnormal TG was lower in $\geq 60$ yrs. than in 44-59 yrs. in males [32]. The MORGAM research in European subjects showed the same abnormal TG trend as that in our study in both genders among 40-49, 50-59 and 6078 yrs. age groups [33]. However, in NHANES (2003-2006) and Framingham Offspring Study [26, 27, 34], the TG disorders continued to rise until aged 60-69 yrs. in both genders. The exact mechanisms regarding age-related TG change between genders have not been clarified yet. Various mechanisms have been proposed to explain the differences.
There is an obvious difference in fat distribution between men and women [35]. One study has suggested that the redistribution of body fat is associated with the TG change in older women [36]. Other evidence suggests that in postmenopausal and premenopausal women, the metabolic changes might be attributed to the different hormonal levels, with the sex hormones being of major importance [37]. In our population, the molecular mechanisms of TG change with age and the role of sex hormone in this process need more laboratory work.

Another thing to be noted is that the average TG levels showed decline tendency recently in some countries, e.g. the US [38, 39], while in China the average TG levels is still increasing $[40,41]$. We think the decrease of TG in males does not just come from the age, but also reflect differences between the subjects. In the current study, the incidence of male TG disorders were positively associated with higher educational level or higher BMI and negatively associated with current smoking status. Socioeconomic status has long been 


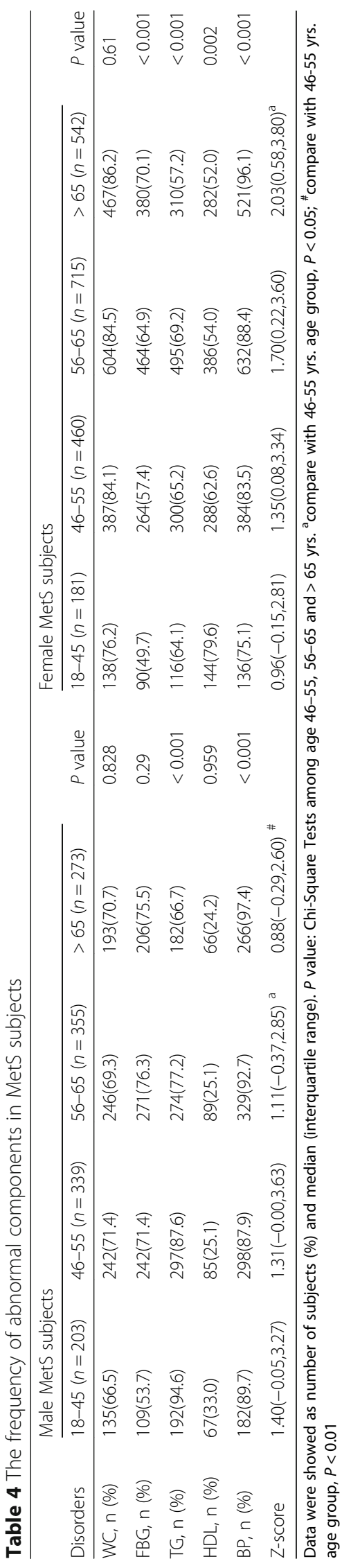




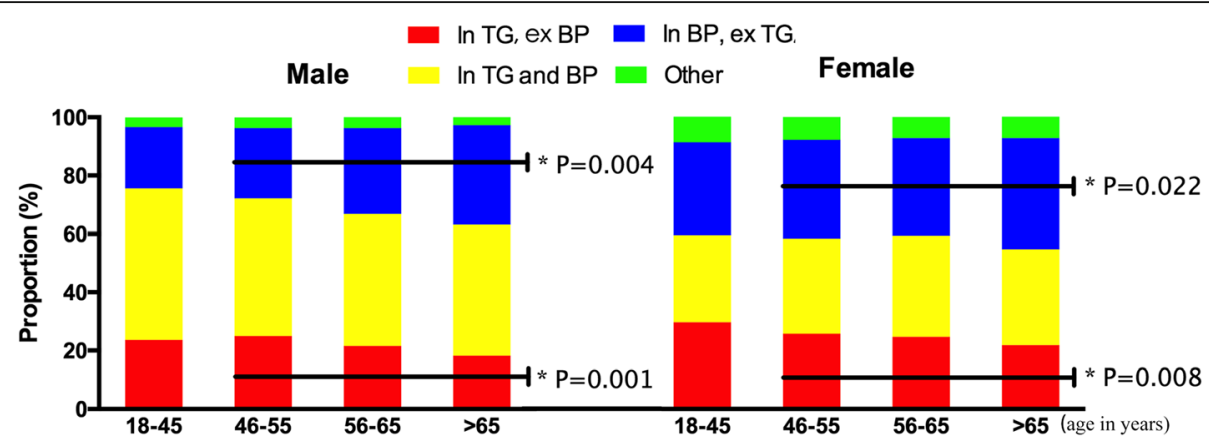

Fig. $\mathbf{2}$ Comparison of various components clustering in all MetS subjects according to gender and age group. In: include, ex: exclude. $P$ value: linear by linear association of Chi-Square tests among 46-55, 56-65 and $>65$ yrs. age group

known to predict higher rates of many chronic diseases and low socioeconomic status may affect women's health to a greater extent [42, 43]. These sociodemographic characteristics may contribute to the decline of TG in older age groups and people with these risk factors should be the important target population of prevention and control of MetS.

Although the prevalence remained stable across age groups, the metabolic disorder spectrum dramatically changed in male MetS subjects, which characterized by less frequent TG disorders and more frequent BP disorders in older age groups. The trends of abnormal BP and BG were noted in several Chinese studies [44] in which abnormal BP became the most common component. This was significantly different from western countries, where abnormal WC remained the most common component $[27,45]$ and BP disorder even decreased [46].

One interesting finding in our analysis was the whole metabolic profile got better in male older age groups. This meant the total disorder degree of five components of MetS, which were equally weighted in the calculation, improved with age. Although the clinical prognostic significance of z-score needs further investigation, at least from the value the five components of MetS got better controlled in older males.

The age-standardized prevalence of metabolic syndrome reported in our study was $21.9 \%$ in men and $22.0 \%$ in women, and there was no statistical difference between genders. Adopting the same diagnostic criteria, the nonstandardized prevalence $33.9 \%$ (31.0\% in men and 36.8\% in women) from the 2010 China Non-communicable Disease Surveillance [20] and 39\% (31.4\% in men and 45.6\% in women) from Rural China obviously overestimated the MetS prevalence in China [17]. Actually, the overall agestandardized prevalence from the China Health and $\mathrm{Nu}-$ trition Survey conducted in 2009 were $21.3 \%$ (20.9\% in males and $21.7 \%$ in females) [16], which was quite close to our results. The standardized prevalence of MetS was even lower in rural area of Northwest China $(15.1 \%, 12.8 \%$ in males and $17.4 \%$ in females) [18]. We think the overall
MetS prevalence of Chinese is lower than the US American (34.3\%) [27], but comparable to Canadians (19.1\%) [45] and other Asian countries [47]. As to gender difference of prevalence, in our study, the higher percentage at early age and lower percentage at old age in males make the overall prevalence equal between genders. Data from the NHANES [28] and the Canadian [45] studies also showed no differences in the overall prevalence of MetS between genders. Whether the similar prevalence between genders is just a transient balance or there are other unknown mechanisms to the equilibrium needs further investigation.

Over the last few decades, China's remarkable economic development has significantly improved people's living standards, including food consumption and nutritional status. The per-capita consumption of proteins steadily increased from 1989 to 2009, especially the proteins from dairy products and processed meat. The intake of oils and fats also increased, but consumption of carbohydrates remained more or less stable. Moreover, fruit and vegetable intake increased significantly over that period [48]. Previous studies have found that excessive caloric intake, processed meat and fried foods could promote the incidence of MetS [49]. However, a high consumption of fruit, vegetables and dairy products is associated with a decreased risk of having MetS. Greater intakes of fruit and vegetables also are associated with lower triglycerides [50]. In our study, gender differences in dietary intake among Chinese adults and their role in the incidence of MetS require further exploration.

There were several limitations in the present study. First, our cross-sectional study only gave information about the prevalence among age groups, not with aging. Therefore, future studies, especially prospective studies are needed to confirm the prevalence difference with aging. Second, the prevalence of MetS and its components would change over time and the diagnostic criteria of MetS were not uniform before 2010, the comparisons between the prevalence need to be explained cautiously. Third, the number of very old ( $>75 \mathrm{yrs}$ ) people in our 
participants was small and they were not divided as another age group. Some previous research showed declined prevalence of MetS in this age group [26], so our results for the oldest age group ( $\geq 65$ yrs) should be interpreted with caution. Fourth, in the logistic analysis of TG disorders, we only collected and analyzed participants' occupation, marital status and educational level. Some important risk factors, such as family income, diet behaviors and physical activity data are not taking into account, so the associations of these factors with TG disorders are not clear.

\section{Conclusions}

In summary, unlike the continuous MetS rise with age in females, the MetS prevalence in males was higher than that in females in 18-45 yrs. age group and remained stable among aged 46 yrs. or older age groups, which could be attributed to the improved TG disorders. The less frequent TG disorder and more frequent BP disorder in old Chinese MetS subjects need more targeted intervention strategies to be carried out to meet the serious challenges of metabolic diseases.

\section{Additional files}

Additional file 1: Table S1. The sociodemographic characteristics by age in both genders (DOCX $22 \mathrm{~kb}$ )

Additional file 2: Table S2. The clustering of various components by age and gender (DOCX $22 \mathrm{~kb}$ )

\section{Abbreviations}

BMI: Body mass index; BP: Blood pressure; CVD: Cardiovascular disease; DM: Diabetes mellitus; FBG: Fasting blood glucose; HDL-C: High-density lipoprotein cholesterol; MetS: Metabolic syndrome; TG: High triglyceride; WC: Waist circumference

\section{Acknowledgments}

We would like to thank the participants of the survey. We are grateful to the village doctors for their efforts in the coordination of the fieldwork. We are also grateful to all the investigators for their contribution to data collection. Thanks should also be expressed to the editor and reviewers of the manuscript for their constructive suggestions.

\section{Fundings}

This study was supported by (1) National Natural Science Foundation of China $(81,270,88581,070,677,81,300,653)$, (2) " 973 " fund by Ministry of Science and Technology in China (2012CB524906) (3) Fund for Clinical Potential Subject 282 Construction of Shanghai JiaoTong University School of Medicine [2014]; (4) Major project of Science and Technology Commission of Shanghai Municipality from Yangtze River Delta epidemiological and intervention studies of environmental pollution and type 2 diabetes (14495810700). (5) Clinical Research Program of 9th People's Hospital, Shanghai Jiao Tong University, School of Medicine (2016).

\section{Availability of data and materials}

The datasets used and analyzed during the current study are not publicly available due to the individual privacy of the patients included in this study, but are available from the corresponding author on reasonable request.

\section{Authors' contributions}

YL designed the study; BJ, YZ, YC, YC, QL, CZ, NW, BH and HZ participated in acquisition of data; $B J$ and $Y L$ evaluated the literature; BJ and YZ undertook the statistical analysis and wrote the first draft of the manuscript. All authors have approved the final manuscript for publication.

\section{Ethics approval and consent to participate}

The study protocol was approved by the Ethics Committee of Shanghai Ninth People's Hospital, Shanghai JiaoTong University School of Medicine. All participants provided written informed consent before data collection.

\section{Competing interests}

The authors declare that they have no competing interests.

\section{Publisher's Note}

Springer Nature remains neutral with regard to jurisdictional claims in published maps and institutional affiliations.

Received: 22 October 2017 Accepted: 16 April 2018

Published online: 20 April 2018

\section{References}

1. Alberti KG, Eckel RH, Grundy SM, Zimmet PZ, Cleeman JI, Donato KA, et al. Harmonizing the metabolic syndrome: a joint interim statement of the international diabetes federation task force on epidemiology and prevention; National Heart, Lung, and Blood Institute; American Heart Association; world heart federation; international atherosclerosis society; and International Association for the Study of obesity. Circulation. 2009;120:1640-5.

2. Wilson PW, D'Agostino RB, Parise H, Sullivan L, Meigs JB. Metabolic syndrome as a precursor of cardiovascular disease and type 2 diabetes mellitus. Circulation. 2005;112:3066-72.

3. Mottillo S, Filion KB, Genest J, Joseph L, Pilote L, Poirier P, et al. The metabolic syndrome and cardiovascular risk a systematic review and metaanalysis. J Am Coll Cardiol. 2010;56:1113-32.

4. Ford ES. The metabolic syndrome and mortality from cardiovascular disease and all-causes: findings from the National Health and nutrition examination survey II mortality study. Atherosclerosis. 2004;173:309-14.

5. Benetos A, Thomas F, Pannier B, Bean K, Jego B, Guize L. All-cause and cardiovascular mortality using the different definitions of metabolic syndrome. Am J Cardiol. 2008;102:188-91.

6. Hu G, Qiao Q, Tuomilehto J, Balkau B, Borch-Johnsen K, Pyorala K, et al. Prevalence of the metabolic syndrome and its relation to all-cause and cardiovascular mortality in nondiabetic European men and women. Arch Intern Med. 2004;164:1066-76.

7. Zheng Y, Stein R, Kwan T, Yu C, Kwan J, Chen SL, et al. Evolving cardiovascular disease prevalence, mortality, risk factors, and the metabolic syndrome in China. Clin Cardiol. 2009;32:491-7.

8. Park YW, Zhu S, Palaniappan L, Heshka S, Carnethon MR, Heymsfield SB. The metabolic syndrome: prevalence and associated risk factor findings in the US population from the third National Health and nutrition examination survey, 1988-1994. Arch Intern Med. 2003;163:427-36.

9. Hildrum B, Mykletun A, Hole T, Midthjell K, Dahl AA. Age-specific prevalence of the metabolic syndrome defined by the international diabetes federation and the National Cholesterol Education Program: the Norwegian HUNT 2 study. BMC Public Health. 2007;7:220.

10. Moore JX, Chaudhary N, Akinyemiju T. Metabolic syndrome prevalence by race/ethnicity and sex in the United States, National Health and nutrition examination survey, 1988-2012. Prev Chronic Dis. 2017;14:E24.

11. Jiang B, Li B, Wang Y, Han B, Wang N, Li Q, et al. The nine-year changes of the incidence and characteristics of metabolic syndrome in China: longitudinal comparisons of the two cross-sectional surveys in a newly formed urban community. Cardiovasc Diabetol. 2016;15:84.

12. Loucks EB, Rehkopf DH, Thurston RC, Kawachi I. Socioeconomic disparities in metabolic syndrome differ by gender: evidence from NHANES III. Ann Epidemiol. 2007;17:19-26.

13. Kang Y, Kim J. Gender difference on the association between dietary patterns and metabolic syndrome in Korean population. Eur J Nutr. 2016;55:2321-30.

14. Du S, Lu B, Zhai F, Popkin BM. A new stage of the nutrition transition in China. Public Health Nutr. 2002;5:169-74. 
15. Ng SW, Norton EC, Popkin BM. Why have physical activity levels declined among Chinese adults? Findings from the 1991-2006 China health and nutrition surveys. Soc Sci Med. 2009;68:1305-14.

16. Xi B, He D, Hu Y, Zhou D. Prevalence of metabolic syndrome and its influencing factors among the Chinese adults: the China health and nutrition survey in 2009. Prev Med. 2013;57:867-71.

17. Yu S, Guo X, Yang H, Zheng L, Sun Y. An update on the prevalence of metabolic syndrome and its associated factors in rural Northeast China. BMC Public Health. 2014;14:877.

18. Zhao $Y$, Yan $H$, Yang $R$, Li Q, Dang S, Wang Y. Prevalence and determinants of metabolic syndrome among adults in a rural area of Northwest China. PLoS One. 2014;9:e91578.

19. Song QB, Zhao Y, Liu YQ, Zhang J, Xin SJ, Dong GH. Sex difference in the prevalence of metabolic syndrome and cardiovascular-related risk factors in urban adults from 33 communities of China: the CHPSNE study. Diab Vasc Dis Res. 2015;12:189-98.

20. Lu J, Wang L, Li M, Xu Y, Jiang Y, Wang W, et al. Metabolic syndrome among adults in China: the 2010 China noncommunicable disease surveillance. J Clin Endocrinol Metab. 2017;102:507-15.

21. Wang N, Wang X, Han B, Li Q, Chen Y, Zhu C, et al. Is exposure to famine in childhood and economic development in adulthood associated with diabetes? J Clin Endocrinol Metab. 2015;100:4514-23.

22. Zhou BF. Cooperative meta-analysis Group of the Working Group on obesity in C. Predictive values of body mass index and waist circumference for risk factors of certain related diseases in Chinese adults-study on optimal cut-off points of body mass index and waist circumference in Chinese adults. Biomed. Environ. Sci. 2002;15:83-96.

23. Alberti KG, Zimmet PZ. Definition, diagnosis and classification of diabetes mellitus and its complications. Part 1: diagnosis and classification of diabetes mellitus provisional report of a WHO consultation. Diabet. Med.: J British Diabe Assoc. 1998;15:539-53.

24. Chalmers J, MacMahon S, Mancia G, Whitworth J, Beilin L, Hansson L, et al. World Health Organization-International Society of Hypertension Guidelines for the management of hypertension. Guidelines subcommittee of the World Health Organization. Clinical and experimental hypertension. 1999. 21:1009-60.

25. Johnson JL, Slentz CA, Houmard JA, Samsa GP, Duscha BD, Aiken LB, et al. Exercise training amount and intensity effects on metabolic syndrome (from studies of a targeted risk reduction intervention through defined exercise). Am J Cardiol. 2007;100:1759-66.

26. Ervin RB. Prevalence of metabolic syndrome among adults 20 years of age and over, by sex, age, race and ethnicity, and body mass index: United States, 2003-2006. Natl Health Stat Rep. 2009:5:1-7.

27. Ford ES, Li C, Zhao G. Prevalence and correlates of metabolic syndrome based on a harmonious definition among adults in the US. Journal of diabetes. 2010;2:180-93.

28. Ford ES, Giles WH, Mokdad AH. Increasing prevalence of the metabolic syndrome among US. adults. Diabetes Care. 2004;27:2444-9.

29. Scuteri A, Laurent S, Cucca F, Cockcroft J, Cunha PG, Manas LR, et al. Metabolic syndrome across Europe: different clusters of risk factors. Eur J Prev Cardiol. 2015;22:486-91.

30. Slagter SN, van Waateringe RP, van Beek AP, van der Klauw MM, Wolffenbuttel BHR, van Vliet-Ostaptchouk JV. Sex, BMI and age differences in metabolic syndrome: the Dutch lifelines cohort study. Endocrine connections. 2017:6:278-88.

31. Al-Daghri NM, Alkharfy KM, Al-Attas OS, Khan N, Alfawaz HA, Alghanim SA, et al. Gender-dependent associations between socioeconomic status and metabolic syndrome: a cross-sectional study in the adult Saudi population. BMC Cardiovasc Disord. 2014;14:51

32. Li JH, Wang LM, Li YC, Bi YF, Jiang Y, Mi SQ, et al. Epidemiologic characteristics of dyslipidemia in Chinese adults 2010. Zhonghua yu fang yi xue za zhi [Chin J Prev Med]. 2012:46:414-8.

33. Vishram JK, Borglykke A, Andreasen AH, Jeppesen J, Ibsen $\mathrm{H}_{\text {, }}$ Jorgensen $T$, et al. Impact of age and gender on the prevalence and prognostic importance of the metabolic syndrome and its components in Europeans. The MORGAM prospective cohort project. PLoS One. 2014;9:e107294.

34. Schaefer EJ, Lamon-Fava S, Cohn SD, Schaefer MM, Ordovas JM, Castelli WP, et al. Effects of age, gender, and menopausal status on plasma low density lipoprotein cholesterol and apolipoprotein B levels in the Framingham offspring study. J Lipid Res. 1994;35:779-92.
35. Freedman DS, Jacobsen SJ, Barboriak JJ, Sobocinski KA, Anderson AJ, Kissebah $\mathrm{AH}$, et al. Body fat distribution and male/female differences in lipids and lipoproteins. Circulation. 1990;81:1498-506.

36. Zamboni M, Armellini F, Harris T, Turcato E, Micciolo R, Bergamo-Andreis IA, et al. Effects of age on body fat distribution and cardiovascular risk factors in women. Am J Clin Nutr. 1997;66:111-5.

37. Peters HW, Westendorp IC, Hak AE, Grobbee DE, Stehouwer CD, Hofman A, et al. Menopausal status and risk factors for cardiovascular disease. J Intern Med. 1999;246:521-8.

38. Carroll M, Kit B, Lacher D. Trends in elevated triglyceride in adults: United States, 2001-2012. NCHS data brief. 2015;198:198.

39. Latifi SM, Karandish M, Shahbazian HB, Chinipardaz R, Sabet A, Pirani N. A survey of the incidence of dyslipidemia and its components in people over 20 years old in Ahvaz: a cohort study 2009-2014. Diabetol Metab Syndr. 2017;11:S751-S754.

40. Li JH, Wang LM, Mi SQ, Zhang M, Li YC, Jiang Y, et al. Awareness rate, treatment rate and control rate of dyslipidemia in Chinese adults, 2010. Zhonghua yu fang yi xue za zhi [Chin J Prev Med]. 2012;46:687-91.

41. Pan L, Yang Z, Wu Y, Yin RX, Liao Y, Wang J, et al. The prevalence, awareness, treatment and control of dyslipidemia among adults in China. Atherosclerosis. 2016;248:2-9.

42. Thurston RC, Kubzansky LD, Kawachi I, Berkman LF. Is the association between socioeconomic position and coronary heart disease stronger in women than in men? Am J Epidemiol. 2005;162:57-65.

43. Ngo AD, Paquet C, Howard NJ, Coffee NT, Adams R, Taylor A, et al. Arealevel socioeconomic characteristics and incidence of metabolic syndrome: a prospective cohort study. BMC Public Health. 2013;13:681.

44. Xu S, Ming J, Yang C, Gao B, Wan Y, Xing Y, et al. Urban, semi-urban and rural difference in the prevalence of metabolic syndrome in Shaanxi province, northwestern China: a population-based survey. BMC Public Health. 2014;14:104.

45. Riediger ND, Clara I. Prevalence of metabolic syndrome in the Canadian adult population. Can. Med. Assoc. J. 2011;183:E1127-34.

46. Beltran-Sanchez H, Harhay MO, Harhay MM, McElligott S. Prevalence and trends of metabolic syndrome in the adult U.S. population, 1999-2010. J Am Coll Cardiol. 2013;62:697-703.

47. Yang JJ, Yoon HS, Lee SA, Choi JY, Song M, Han S, et al. Metabolic syndrome and sex-specific socio-economic disparities in childhood and adulthood: the Korea National Health and nutrition examination surveys. Diabetic medicine : a journal of the British Diabetic Association. 2014; 31:1399-409.

48. Zhang B, Zhai FY, Du SF, Popkin BM. The China health and nutrition survey, 1989-2011. Obesity review. 2014;15(Suppl 1):2-7.

49. Lutsey PL, Steffen LM, Stevens J. Dietary intake and the development of the metabolic syndrome: the atherosclerosis risk in communities study. Circulation. 2008;117:754-61.

50. Panagiotakos DB, Polychronopoulos E. The role of Mediterranean diet in the epidemiology of metabolic syndrome; converting epidemiology to clinical practice. Lipids Health Dis. 2005;4:7.

\section{Ready to submit your research? Choose BMC and benefit from:}

- fast, convenient online submission

- thorough peer review by experienced researchers in your field

- rapid publication on acceptance

- support for research data, including large and complex data types

- gold Open Access which fosters wider collaboration and increased citations

- maximum visibility for your research: over $100 \mathrm{M}$ website views per year

At BMC, research is always in progress.

Learn more biomedcentral.com/submissions 